\title{
The Zadko Telescope: A Southern Hemisphere Telescope for Optical Transient Searches, Multi-Messenger Astronomy and Education
}

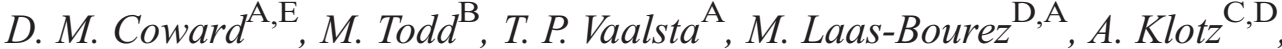 \\ A. Imerito ${ }^{\mathrm{A}}$, L. Yan $^{\mathrm{A}}$, P. Luckas ${ }^{\mathrm{A}}$, A. B. Fletcher ${ }^{\mathrm{A}}$, M. G. Zadnik ${ }^{\mathrm{B}}$, \\ R. R. Burman ${ }^{\mathrm{A}}$, D. G. Blair ${ }^{\mathrm{A}}$, J. Zadko ${ }^{\mathrm{A}}$, M. Boër ${ }^{\mathrm{D}}$, P. Thierry ${ }^{\mathrm{C}}$, E. J. Howell ${ }^{\mathrm{A}}$, \\ S. Gordon ${ }^{\mathrm{A}}$, A. Ahmat ${ }^{\mathrm{A}}$, J. A. Moore ${ }^{\mathrm{A}}$, and K. Frost ${ }^{\mathrm{A}}$ \\ A School of Physics, M013, The University of Western Australia, 35 Stirling Hwy, \\ Crawley, WA 6009, Australia \\ ${ }^{B}$ Department of Imaging and Applied Physics, Bldg 301, Curtin University of Technology, \\ Kent St, Bentley, WA 6102, Australia \\ ${ }^{C}$ CESR, Observatoire Midi-Pyrénées, CNRS, Université de Toulouse, BP 44346, \\ F-31028 Toulouse Cedex 4, France \\ ${ }^{D}$ Observatoire de Haute-Provence, F-04870 Saint Michel l'Observatoire, France \\ ${ }^{\mathrm{E}}$ Corresponding author. Email: coward@physics.uwa.edu.au
}

Received 2009 October 19, accepted 2010 June 9

\begin{abstract}
The new $1 \mathrm{~m} \mathrm{f} / 4$ fast-slew Zadko Telescope was installed in June 2008 about 70 km north of Perth, Western Australia. It is the only metre-class optical facility at this southern latitude between the east coast of Australia and South Africa, and can rapidly image optical transients at a longitude not monitored by other similar facilities. We report on first imaging tests of a pilot program of minor planet searches, and Target of Opportunity observations triggered by the Swift satellite. In 12 months, 6 gamma-ray burst afterglows were detected, with estimated magnitudes; two of them, GRB $090205(z=4.65)$ and GRB $090516(z=4.11)$, are among the most distant optical transients imaged by an Australian telescope. Many asteroids were observed in a systematic 3-month search. In September 2009, an automatic telescope control system was installed, which will be used to link the facility to a global robotic telescope network; future targets will include fast optical transients triggered by high-energy satellites, radio transient detections, and LIGO gravitational wave candidate events. We also outline the importance of the facility as a potential tool for education, training, and public outreach.
\end{abstract}

Keywords: instrumentation: miscellaneous — gamma rays: bursts — minor planets, asteroids — telescopes

\section{Introduction}

The Universe is teeming with fleeting transients, visible across the entire electromagnetic spectrum. In the optical, some last for only a few seconds, while others vary in brightness over years. Sources classed as transient include blazars, cataclysmic variable stars, stellar flares, supernovae, and hypernovae; many of the recently discovered sources are not easily explained.

Some transients, such as gamma-ray bursts (GRBs), have complex emissions spanning the gamma-ray, X-ray and optical bands, and are possibly also strong gravitational wave and neutrino sources. In 'multi-messenger' astronomy (see Sathyaprakash \& Schutz 2009), coordinated observations of the different kinds of radiation and information carriers emitted by such sources offers the opportunity to probe the physics of these exotic transients, by studying their diverse emissions over a range of energies and timescales, from seconds to days.

There are new and upcoming facilities which are expected to discover a huge number of transient objects — these include large optical and radio facilities, space-based detectors, and the next generation of gravitational wave and neutrino observatories. The $1 \mathrm{~m}$ Zadko Telescope, with its $1^{\circ}$ field of view, is perfectly poised to study the transient sky, by following up and monitoring the many transients that will be detected by these facilities in the coming decade.

The study of transient optical sources that vary on timescales of seconds to hours is an exciting and growing field, boosted by the widespread use of small to medium, rapid-response automated telescopes. Many of the smaller instruments are being networked to coordinate observations to allow near-continuous monitoring of rapidly fading (or flaring) optical transients.

The Zadko Telescope (ZT), shown in Figure 1, is just starting first operations as a networked robotic observatory. Made possible by a philanthropic donation to the University of Western Australia (UWA), it is a new resource for research, training, and science education. It is colocated with a science and astronomy outreach 


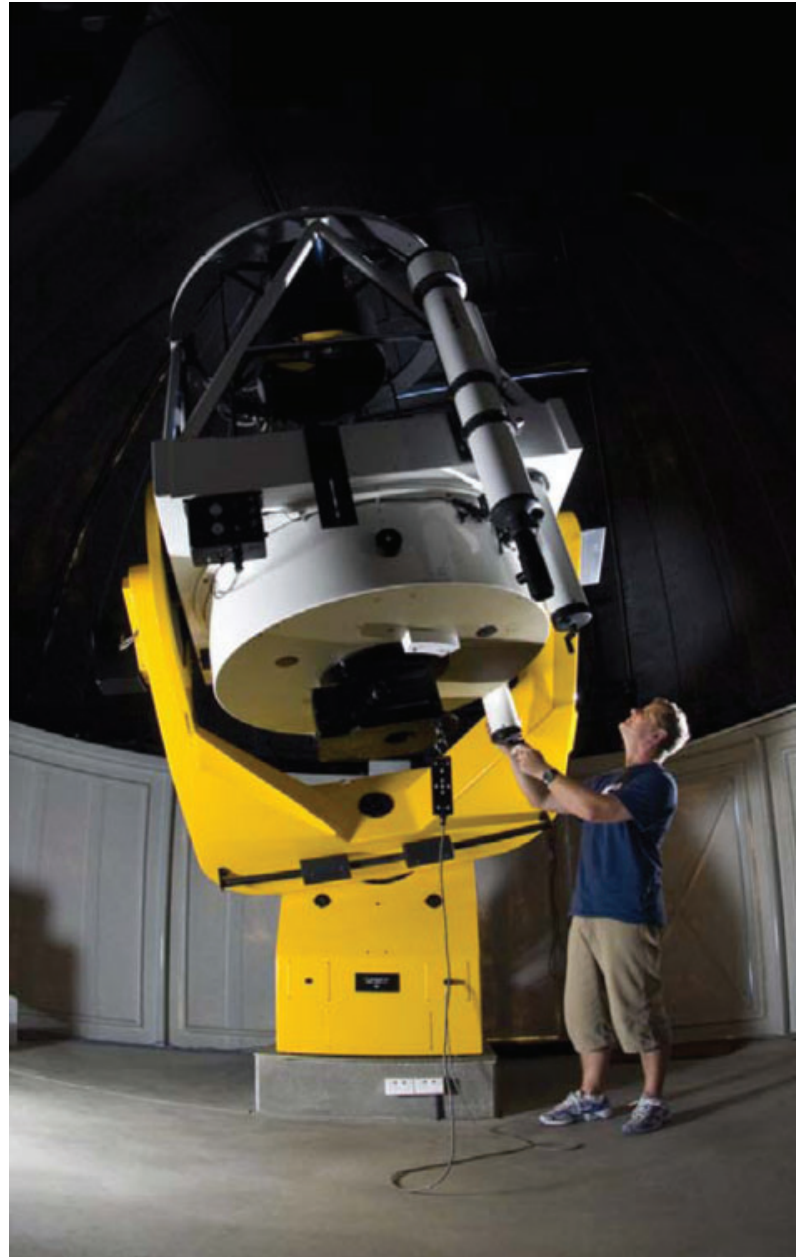

Figure 1 Zadko Telescope: a new $1 \mathrm{~m} \mathrm{f} / 4$ Cassegrain reflector, located $67 \mathrm{~km}$ north of Perth. Built by DFM Engineering, Inc., and donated to UWA by resource company Claire Energy. It was opened by Australia's Chief Scientist on 1 April 2009.

facility, and with the Australian International Gravitational Observatory (AIGO).

The leaders of Télescopes à Action Rapide pour les Objets Transitoires (TAROT, ${ }^{1}$ the French robotic telescope network; Klotz et al. 2008a) recognised that the ZT is uniquely located in a region devoid of similar facilities. This led to an ongoing partnership to robotise the facility for optical transient science, using the TAROT robotic observatory control system. The joint science capability of the ZT will be substantially extended by this partnership in two ways: firstly, the ZT will provide deeper observations, so it can follow up on transients fainter than TAROT can detect; secondly, it will increase the overall detection rate, due to its wide longitudinal separation from the Chilean TAROT site.

The ZT, in partnership with other facilities, such as TAROT and SkyMapper, can make valuable contributions to frontier multi-messenger astronomy, including searches for optical counterparts of radio, X-ray, and LIGO gravitational wave (GW) transients.
The $\mathrm{LIGO}^{2}$ and $\mathrm{VIRGO}^{3}$ interferometers are now entering a sensitivity regime where GW emissions from coalescing compact binaries should be detectable out to 10s of Mpc; the next-generation Advanced GW detectors will expand this horizon out to 100s of Mpc (Abadie et al. 2010). This presents an enormous opportunity to combine optical transient searches with GW burst searches. From early 2010 , the ZT is being employed by LIGO as a test facility for optical follow-ups of large error boxes $\left(\approx 10 \mathrm{deg}^{2}\right)$. The first tests and methods of this pilot program will be reported in a later publication.

The ZT has already allowed scientists to engage with the broader community on several research projects that have captured the public imagination. Furthermore, the ZT has opened up new research opportunities that have the potential to contribute to Australia's already important role in optical transient astronomy. In this paper, we describe the status of the facility, and its planned role in several current and emerging transient astronomy science programs.

\section{The Zadko Telescope}

The $\mathrm{ZT}^{4}$ is a new $1 \mathrm{~m} \mathrm{f} / 4$ Cassegrain telescope, built by DFM Engineering, Inc., ${ }^{5}$ and situated in the state of Western Australia at longitude $115^{\circ} 42^{\prime} 49^{\prime \prime}$ E, latitude $31^{\circ} 21^{\prime} 24^{\prime \prime} \mathrm{S}$, and altitude $47 \mathrm{~m}$ above sea level. Located $67 \mathrm{~km}$ north of Perth, the site has a typical 'Californian' climate - hot, dry summers with mostly clear skies, and mild, wet winters, with a mean of 83 clear days per year. The closest towns are each $19 \mathrm{~km}$ away, with small populations ( $1628 \& 531$ in the 2006 Census); the site is not strongly affected by man-made lighting.

The ZT is an equatorial, fork-mounted telescope with a primary mirror clear aperture of $1007.0 \mathrm{~mm}$, and a system focal length of $4038.6 \mathrm{~mm}$. Its fast optics have a low f-ratio, and a flat and wide field of view. It uses state-ofthe-art friction drives to achieve high stiffness and excellent tracking on both axes. The fork mount provides for a convenient and compact telescope, able to carry a maximum instrument load of $200 \mathrm{~kg}$, and requiring minimal counterweights for fast dynamic response - with a maximum slew speed of $3.0^{\circ} \mathrm{s}^{-1}$. The observatory's Sirius dome rotates at $3.7^{\circ} \mathrm{s}^{-1}$ on average.

The primary and secondary mirrors are spaced with Invar (ArcelorMittal: Luxembourg) rods to minimise focus shift with temperature. The carbon fibre technology within the focus housing provides additional thermal compensation of the optical spacing. ${ }^{6}$ For a slowly changing temperature, the optical assembly has an essentially zero temperature coefficient.

\footnotetext{
${ }^{2}$ http: / /www.ligo.caltech.edu.

${ }^{3}$ http: / / www. virgo.infn.it.

${ }^{4}$ http: / /www. zt.science. uwa. edu. au.

${ }^{5}$ http://www. dfmengineering.com.

${ }^{6}$ This focus housing delivered by DFM Engineering, Inc., is the first based on a new carbon fibre design.
} 
The ZT is mounted on a vibration-isolated concrete pier, and housed in a $6.7 \mathrm{~m}$ diameter fibreglass dome. Mechanical vibrations transferred from the observatory floor to the mount do not exceeded 1 arcsec, and are damped within $1 \mathrm{~s}$. The dome has two shutters: the upper is driven by electric motor, and the lower is a hydraulically controlled drawbridge; both are powered by battery, which is recharged by a solar panel. The dome is interfaced with the ZT using the AStronomy Common Object Model (ASCOM) protocol, and is controlled via the French Robotic Observatory Control System, installed in September 2009 (see Section 3).

\subsection{Optical Design}

The ZT optical design is a hybrid related to the traditional Ritchey-Chrétien (RC), but with a small, thin, 4th-order refracting field corrector/flattener. The two mirrors form a modified RC system, but are significantly more aspheric - especially the secondary mirror. The optical design also incorporates the effects of the glass in the filter wheel and CCD vacuum window.

In this design, incident light is reflected by a lightweight f/2.3 primary mirror of $1007.0 \mathrm{~mm}$ diameter (clear aperture), an f/4.9 secondary mirror of $469.9 \mathrm{~mm}$ diameter, and passes through the very fast and thin $6.35 \mathrm{~mm}$ thick 4th-order Schmidt corrector/flattener plate of $236.2 \mathrm{~mm}$ diameter, placed $126.2 \mathrm{~mm}$ above the primary mirror surface. The resulting system focal ratio is $\mathrm{f} / 4.0105$ at the Cassegrain focus, which is nominally $467.9 \mathrm{~mm}$ below the primary mirror surface.

Focusing is achieved at the secondary mirror housing which allows $77 \mathrm{~mm}$ of back focus travel. When properly focused, $80 \%$ of the incident light falls within 0.6 arcsec (in vacuo). The ZT is designed to image on detectors as wide as $99 \mathrm{~mm}$ in diameter, giving an unvignetted $1.4^{\circ}$ field of view (FOV), and the image spot size nominally remains $<1$ arcsec at a deviation of $0.65^{\circ}$ off-axis.

\subsection{Low-noise CCD Imager}

The main ZT imaging detector is the iKon- $\mathrm{L}$ with a DW436-BV back-illuminated sensor, built by Andor Technology PLC. ${ }^{7}$ This is a large-area, low-noise, thermoelectrically-cooled CCD with $2048 \times 2048$ $13.5 \mu \mathrm{m}$ square pixels, corresponding to $0.69 \mathrm{arcsec}$ at the ZT f/4.0 Cassegrain focus; the CCD FOV is $23^{\prime} .6 \times 23^{\prime} .6$. The pixel well-depth is $\approx 100000 e^{-1}$, and the response is linear up to $\approx 80000 e^{-1}$, with a maximum deviation from linearity of $1 \%$. The CCD frame is usually read out at $500 \mathrm{kHz}$; this takes $8 \mathrm{~s}$, with the data stored in an $8 \mathrm{MB}$ FITS file. The readout noise is $\approx 6.5 e^{-1} \mathrm{pix}^{-1}$, with a gain $g \approx 2 e^{-1} \mathrm{ADU}^{-1}$. A short (10 s) dark frame exposure has a

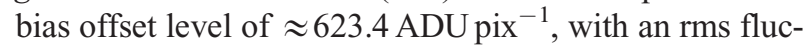
tuation $\approx 3.6 \mathrm{ADU} \mathrm{pix}^{-1}$, comparable to the readout noise. At an operating temperature of $-50^{\circ} \mathrm{C}$, the dark current is typically $\approx 0.030 e^{-} \mathrm{pix}^{-1} \mathrm{~s}^{-1}$.

\footnotetext{
7 http: / / www . andor.com
}

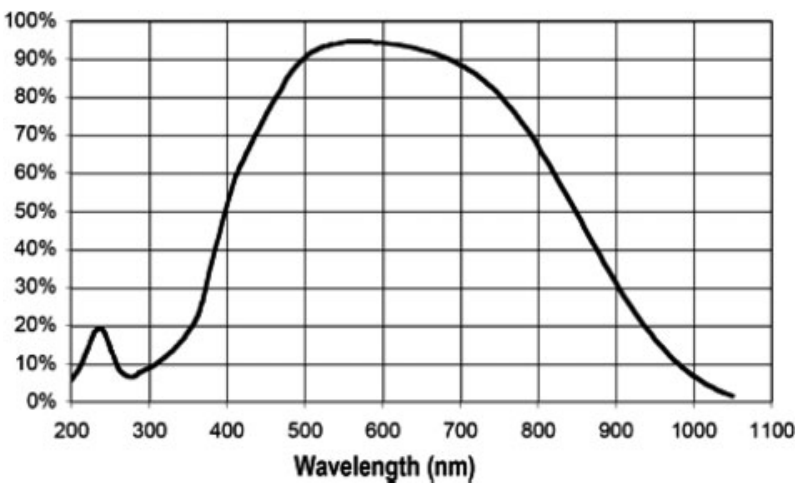

Figure 2 Wavelength sensitivity (QE) of the iKon-L DW436-BV CCD camera (Andor Technology 2007).

The iKon-L sensor is currently used without filters and has a nominal peak quantum efficiency (QE) of $95 \%$ near $5700 \AA$ (in the $V$-band - see Figure 2 ). The effective area of the ZT with unfiltered Andor CCD is proportional to their combined spectral response, which peaks at $A_{\mathrm{EFF}}=3371 \mathrm{~cm}^{2}$ at $\lambda_{\mathrm{p}}=5685 \AA$, with a mean wavelength $\lambda_{\mathrm{m}}=6225 \AA$, and full-width at half-maximum $\Delta \lambda_{\text {FWHM }}=4336 \AA$; the overall response is like a 'wide-V/R' band, with relatively low IR and UV sensitivity.

Assuming Vega's mean spectral irradiance from 2000 to $12000 \AA$ to be $S_{0} \approx 2.58 \times 10^{-9} \mathrm{erg} \mathrm{cm}^{-2} \mathrm{~s}^{-1} \AA^{-1}$, we estimate the zero-point flux ${ }^{8}$ detected by the unfiltered ZT system to be $F_{0} \approx 6.21 \times 10^{-6} \mathrm{erg} \mathrm{cm}^{-2} \mathrm{~s}^{-1}$. Integrating over area, the zero-point power detected is $P_{0} \approx 4.95 \times$ $10^{-2} \mathrm{erg} \mathrm{s}^{-1}$, corresponding to a photoelectron rate of $\Phi_{0} \approx 1.33 \times 10^{10} \mathrm{~s}^{-1}$, and to a zero-point magnitude $m_{0}=24.56 \pm 0.16$; the effective wavelength of Vega is $\lambda_{0} \approx 5332 \AA$ in this system.

Stellar aperture photometry on LIGO trigger widefield mosaic frames taken on 25 July 2009 gave an estimated zero-point of $m_{0}=24.41$ on the USNO-A2.0 magnitude scale, consistent with our preliminary theoretical estimate.

\section{Robotic Observatory Control System}

As part of the TAROT collaboration, the ZT is being robotised so as to be similar to the existing TAROT robotic telescopes (Klotz et al. 2008a). The ultimate aim is to make the ZT a node of the TAROT network, enabling much larger coverage of the transient sky for participation in several frontier optical transient science projects. The centralised system at the core of this network is a cluster of database servers known as the Coordination et Analyse des Données des Observatoires Robotiques (CADOR see Bourez-Laas et al. 2008; Klotz et al. 2008b).

Control of the observatory and initial processing of astronomical images is performed by the TAROT robotic telescope software system. This suite of independently

\footnotetext{
${ }^{8}$ The zero-point flux detected by a telescope system is that from an unextincted standard star of zero magnitude, e.g. Vega (see Zombeck 1990).
} 


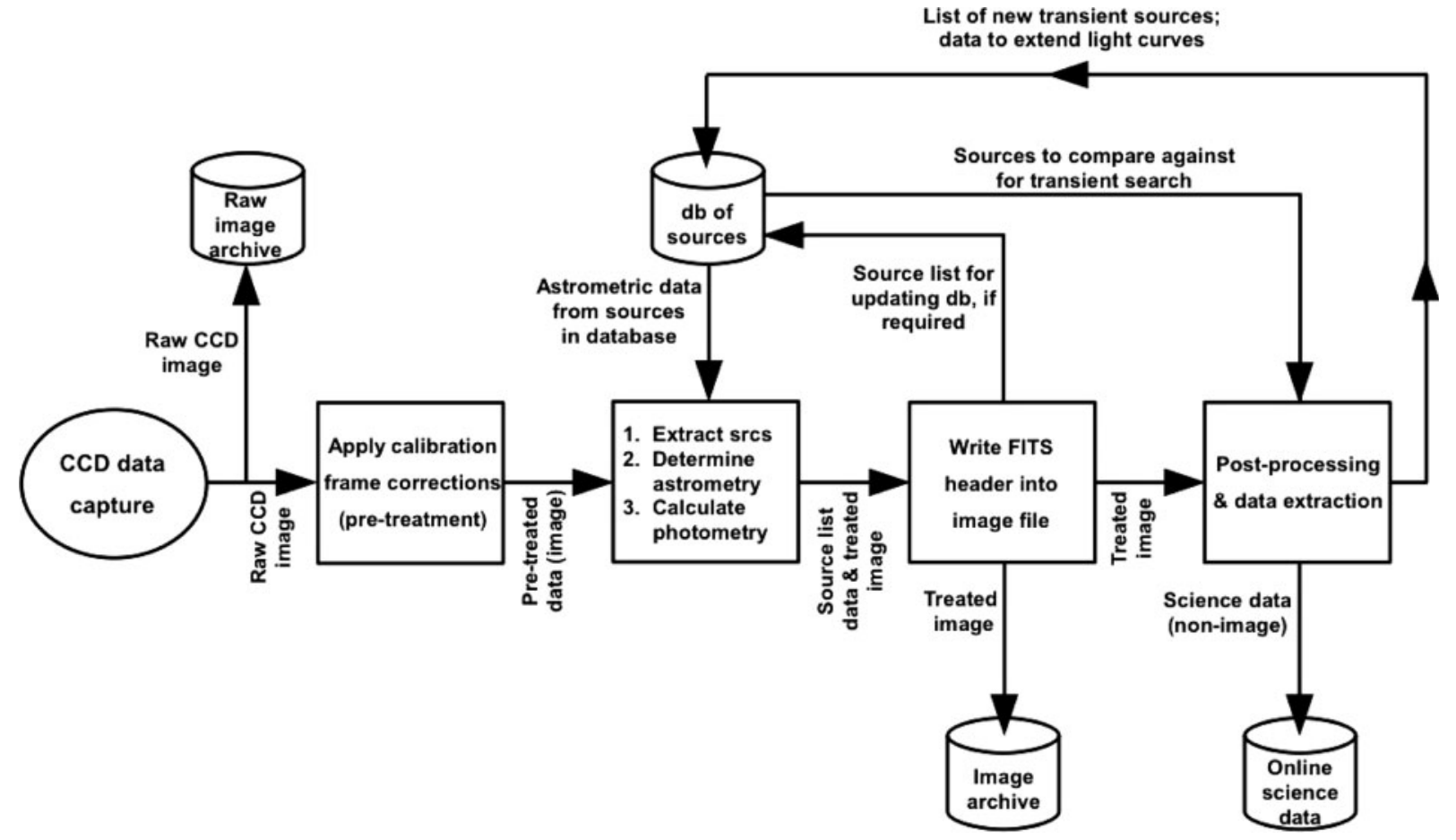

Figure 3 Zadko Telescope automated data processing pipeline.

running programs comprises two main components: AudeLA ${ }^{9}$ and the Robotic Observatory Software (ROS). There is also 3rd-party software (mostly device drivers) for the mechanical and ancillary systems of the observatory (i.e. telescope, dome, dome interior \& weather station). For the ZT, the DFM-supplied Telescope Control System (TCS) is interfaced to ROS via the ASCOM $^{10}$ protocol.

AudeLA is written mainly in $\mathrm{C} / \mathrm{C}++$, and contains lower-level routines than ROS (which is scripted in $\mathrm{Tcl} /$ Tk). The AudeLA routines can be used by an operator to directly control the telescope/camera combination, and to process images. Alternatively, AudeLA can be driven by user-created Tcl/Tk scripts, or by other high-level control software - such as ROS - to create a fully robotic observatory. The following are the main functions of the robotic observatory software:

1. Create a prioritised observation schedule for the night. Observing requests can be made at any time; the queue is dynamically recalculated whenever such changes occur.

2. Conduct telescope observations according to the queued observing requests and priority interrupts (e.g. from GRBs).

3. Perform CCD camera operations: data capture and transfer to disk.

\footnotetext{
9 http: / / www . audela.org/english_audela.php.

${ }^{10}$ http: / / ascom-standards.org. ASCOM is a widely available interface standard for freeware device drivers commonly used in astronomical instruments, e.g. telescopes, cameras, focusers, and domes.
}

4. Preprocess raw images.

5. Monitor environmental conditions, and activate appropriate software to respond to non-benign changes (e.g. strong wind, rain, high temperature or humidity).

6. Operate Webcam for remote monitoring of observatory.

7. Enable data transfer to/from remote locations.

8. Maintain operational $\log$ files.

Many of the above functions rely on AudeLA's routines to perform the operations (e.g. telescope, dome \& camera control, image preprocessing — see Figure 3). After acquiring a raw image from the telescope, which is stored locally on disk, a standard set of processing operations is then performed on a copy of each image. Calibration frame corrections (bias, dark, and flats) are applied. Cosmic ray artefacts are removed. Mirror transformations are applied to put north at the top of the image, and east to the left. Stars are identified and catalogued by SExtractor (Bertin \& Arnouts 1996). Astrometric calibration is then performed (e.g. using the USNO-A2.0 catalogue), followed by photometric determinations. The image file FITS header is written, and the resulting processed file is saved to disk. The CADOR database is also updated with the new sources measured by SExtractor. Postprocessing and data extraction are then done. The observer can specify particular operations to be applied to the image by the automated pipeline (e.g. checking sources for transient events). The processed images and any science products (e.g. light curves) are then made available for the observer to download via the Web. JPEG images are also produced, for Web page rendering. 
The TAROT control system was installed in September 2009, and will be tested in 2010. This robotic commissioning period will also see the installation of new environmental monitoring hardware to ensure that the system is robust. Also, the dome shutter hardware will be modified to reduce the risk of mechanical failure, and to ensure reliable software control. We plan to start science programs in full robotic mode in 2010.

\section{Pilot Science Program}

In 2009 the telescope and imager performance were tested, under different observing conditions, with a pilot science program in optical transient detection. These first tests served two purposes: they provided the first sensitivity limits of the optical and imaging system, and the first photometric data obtained from optical transient observations.

\subsection{ZT Imaging Sensitivity}

A field of faint standard stars centred at RA(J2000) $16^{h} 11^{m} 04^{s}$, Dec.(J2000) + 14 $56^{\prime} 36^{\prime \prime}$ (Saha et al. 2005) was imaged with integration times ranging from 15 to $600 \mathrm{~s}$, from 20 April to 29 May 2009. Stars in the images were detected using a $2 \sigma$ threshold (i.e. $2 \times$ rms noise). Measured stellar FWHMs ranged from 1 to 6 arcsec; varying from 1 to 3.5 arcsec at zenith, and 3.5 to 6 arcsec at $45^{\circ}$ elevation. However, these may tend to overpredict the atmospheric seeing due to problems with collimation and focusing (a good focus cannot be found over the broad ZT bandwidth).

For these first tests the limiting magnitude of the ZT, with the unfiltered Andor camera, was estimated to be $20.5 \pm 0.1$ for integration times of 300 to 600 s under $40 \%$ Moon illumination and with average seeing. With no Moon the system is sensitive to sources with $R \approx 21$ for a single $180 \mathrm{~s}$ exposure. A more typical sensitivity for a $2 \times 180$ s exposure is $R \approx 20$ for a star $90^{\circ}$ from the Moon (at $38 \%$ phase; stellar image $\Theta_{\mathrm{FWHM}}=4.5 \mathrm{arcsec}$ ). Imaging Target of Opportunity transients showed that objects with $m \approx 23$ are marginally detectable (SNR $\approx 3$ ) using deeper exposures in favourable observing conditions (i.e. good seeing, dark sky), as demonstrated by the GRB imaging described in Section 4.3.

Our detailed theoretical calculations of the final SNR - after careful sky subtraction and averaging of calibrated CCD frames - suggest the following unextincted limiting magnitudes for unresolved sources detected (with $\mathrm{SNR}=5$; image $\theta_{\mathrm{FWHM}}=2$ arcsec) by the unfiltered ZT system: $m \approx 24.1$ (23.8) for a $10 \times 100 \mathrm{~s}$ stacked integration at zenith angle $z=0^{\circ}\left(60^{\circ}\right)$ in New Moon; $m \approx 23.0$ (22.7) for a $10 \times 100$ s stacked integration at zenith angle $z=0^{\circ}\left(60^{\circ}\right)$ in Full Moon. In these estimates, typical values of the atmospheric extinction coefficient (see Barbieri 2007) and sky brightness (see McLean 2008) were assumed in the standard UBVRI filter bandpasses.

On the 25 July 2009 a minimum sky brightness of $m \leq 21.16 \pm 0.04 \mathrm{mag} \operatorname{arcsec}^{-2}$ was measured (Moon

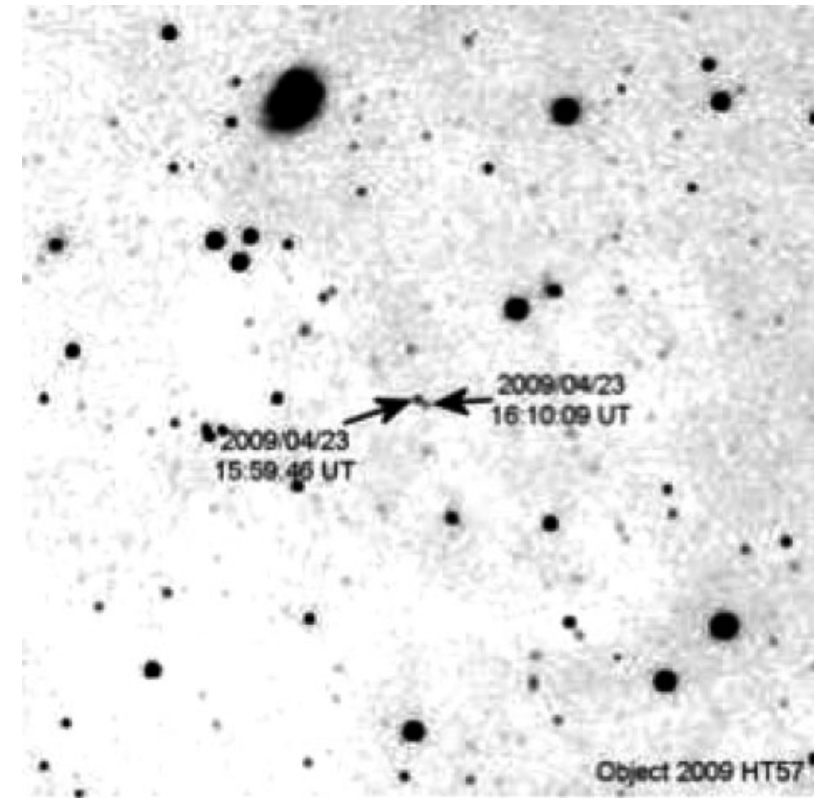

Figure 42009 HT57 image, showing the change in position of this minor planet relative to background stars. Arrows indicate position at noted times. Movement is apparent even over a few minutes.

at $5.2^{\circ}$ elevation, $m=-11,16 \%$ phase, with scattered clouds present).

\subsection{Minor Planet Searches}

In March 2009 a pilot minor planet search program started with observations of selected known Near-Earth Objects (NEOs). Using the Minor Planet Center (MPC) web pages, for both bright and faint objects, ${ }^{11}$ a shortlist of Near-Earth Asteroids (NEAs), with magnitudes in the 18-22.5 range, was compiled for observations in March and April 2009; selection was based on considerations of the number of previous observations, on information from the MPC urging further observations, and on the capability of the ZT to detect faint objects.

The positions of these selected targets were obtained using the Minor Planet Ephemeris Service ${ }^{12}$ provided by the MPC. This service also advises which targets require priority observations, enabling a subset of 4-6 objects to be selected for each observing session. Three images of each target were obtained using $300 \mathrm{~s}$ exposures, followed by a second set of three images $1 \mathrm{~h}$ later. The IRIS astronomical software package ${ }^{13}$ was used for image preprocessing, astrometric, and photometric determinations. The resulting images show the object shifting its position relative to background stars (see Figure 4). These results were subsequently submitted to the MPC to help refine orbit solutions for use in future searches.

\footnotetext{
11 www . cfa.harvard. edu/iau/NEO/TheNEOPage. html.

12 www. cfa.harvard. edu/iau/MPEph/MPEph.html.

13 http: / / www . astrosurf.com/buil/us/iris/iris.htm.
} 
Table 1. Some asteroids found using the ZT (2009)

\begin{tabular}{|c|c|c|c|}
\hline $\begin{array}{l}2009 \mathrm{ZT} \\
\text { designation }\end{array}$ & $\begin{array}{l}2009 \text { ZT } \\
\text { obs. date }\end{array}$ & $\begin{array}{l}\text { Previous } \\
\text { obs. years }\end{array}$ & $\begin{array}{l}2010 \mathrm{Feb} \\
\text { status }\end{array}$ \\
\hline $2009 \mathrm{FH}^{1} 9^{\mathrm{A}}$ & Mar 18 & \multirow{6}{*}{2004,2007} & Arc \\
\hline $2009 \mathrm{FK} 30^{\mathrm{B}}$ & Mar 18 & & Known \\
\hline 2009 FL30 & Mar 21 & & $\operatorname{Arc}^{E}$ \\
\hline 2009 GY02 & Apr 2 & & Arc \\
\hline 2009 GZ02 & Apr 2 & & Arc \\
\hline 2009 FN57 & Mar 27 & & Arc \\
\hline $2009 \mathrm{HT}_{57}{ }^{\mathrm{C}}$ & Apr 20 & \multirow[t]{3}{*}{2002,2005} & Known \\
\hline 2009 FN59 & Mar 27 & & $\operatorname{Lost}^{\mathrm{F}}$ \\
\hline 2009 HY81 & Apr 20 & & Arc \\
\hline $2009 \mathrm{HZ} 1^{\mathrm{D}}$ & Apr 23 & \multirow[t]{3}{*}{ 1999-2009 } & Known \\
\hline 2009 JW12 & May 14 & & Lost \\
\hline 2009 KM22 & May 24 & & Lost \\
\hline
\end{tabular}

A 2009 FH19 is not $30980=1995$ QU3, as prev. claimed by Palomar, and AstDyS database.

${ }^{B} 2009$ FK 30 linked by MPC to 2007 UY14 previously found in 2004 by Apache Point/SDSS survey.

C 2009 HT 57 linked by MPC to 2005 MH16 previously found in 2002 by Palomar/NEAT survey.

D 2009 HZ81 previously found in 1999 by Palomar.

${ }^{\mathrm{E}}$ Orbital elements, derived from a limited arc, are uncertain but predictions are still possible.

${ }^{\mathrm{F}}$ Orbital elements are too uncertain to make any further reliable predictions.

In this program 12 asteroids were found by chance (see Table 1). As of February 2010 at least three of these have since been identified ${ }^{14}$ to be recoveries of known asteroids previously discovered in other programs. Whether any of the remaining nine asteroids are 'new' discoveries (i.e. for which reliable orbital elements have never been determined) is unknown; three of these nine have since been lost, and six have uncertain elements. For the nine objects with known elements, the semi-major axes range from 1.89 to $3.17 \mathrm{AU}$, which suggests that they are mainbelt asteroids.

\subsection{Optical Transient Searches}

TAROT employs two fully robotic $0.25 \mathrm{~m}$ telescope systems located in Calern (France) and La Silla (Chile), mainly for the rapid follow-up of GRBs. GRBs are highenergy transients, lasting from milliseconds to hundreds of seconds. Observations of these emissions provides a probe of the extreme physics associated with the largest explosions in the Universe. Optical flares have been observed by TAROT and other telescopes, in some cases tens of seconds after the trigger, allowing new insight into the prompt emission regime of GRBs (Klotz et al. 2009).

The record of 'missed' GRB optical afterglow observations provides insight into the impact that the $\mathrm{ZT}$ will have on obtaining photometry on bursts at negative declinations. In the following we show how the historical

\footnotetext{
14 http: / / www. minorplanetcenter.org/iau/mpc.html. http://hamilton.dm.unipi.it/astdys/.

http: / / www. hohmanntransfer.com/about.htm.
}

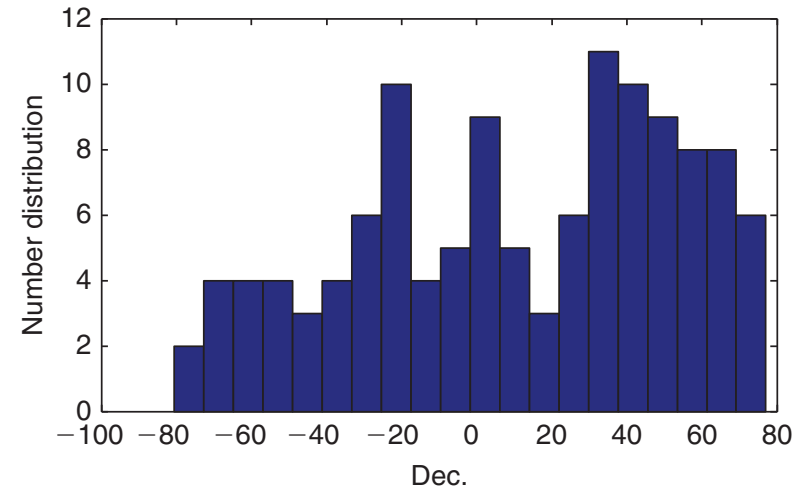

Figure 5 Observed GRB optical afterglow distribution in declination.

record of satellite-triggered optical transient observations has revealed a strong bias in the number of GRB afterglows observed at different declinations.

About half of the observations triggered and localised by the Swift satellite have not been observed with any optical emission, despite deep follow-up observations. To fully capitalise on the positional information from Swift, and other satellite detectors, requires follow-up by optical telescopes of as many triggers as possible. As the continental land mass is strongly biased to the northern hemisphere, most rapid-response telescopes engaged in optical transient science are located in Europe, Asia, and North America. This has resulted in a deficit of GRB optical afterglow (OA) science at declinations south of $-60^{\circ}$. Figure 5 highlights the drop in GRB OA follow-up in the southern hemisphere, by plotting the distribution of GRB OA discoveries with declination. Without OA detections, redshifts cannot be secured to these bursts. Coward et al. (2008) showed how such selection effects can modify the observed spatial distribution of GRB optical properties.

It is clear from this distribution that a niche role exists for metre-class telescopes in Australia, including the ZT, to help fill the GRB OA (and other transient) deficit at negative declinations.

After installing the Andor camera on the $\mathrm{ZT}$ in September 2008, a Target of Opportunity (ToO) pilot program was started, aimed at GRB OAs. Even though the facility was not enabled for robotic response when this program started, ${ }^{15}$ the communication infrastructure was in place to receive external triggers, and to manually intervene in any scheduled observing. Just weeks after installing the camera, the ZT was imaging Swift satellitetriggered GRB optical afterglows reported in online circulars of the GRB Coordinates Network (GCN). ${ }^{16}$ Below, we summarise these pilot observations (also see Table 2):

\footnotetext{
${ }^{15}$ In 2009 September, the French ROS system was installed, enabling rapid response to GRB satellite triggers; this system will be tested over the following year.

${ }^{16}$ http: //gen.gsfc. nasa.gov.
} 
Table 2. Z ZT GRB ToO observations (2008-2009)

\begin{tabular}{llll}
\hline GRB number & $\begin{array}{l}\text { ZT estim. } \\
\text { magnitude }\end{array}$ & $z$ & ZT obs. report \\
\hline GRB 081118 & $20.9 \pm 0.5$ & 2.58 & GCN 8675 \\
GRB 090205 & $23.2 \pm 0.3$ & 4.65 & GCN 8976 \\
GRB 090313 & $18.0 \rightarrow 18.8$ & 3.38 & GCN 8996 \\
GRB 090509 & $>17.6^{\mathrm{C}}$ & & GCN 9363 \\
GRB 090516 & $18.9 \rightarrow 20.3$ & 4.11 & GCN 9380 \\
GRB 090927 $^{\mathrm{B}}$ & $19.8 \rightarrow 20.0^{\mathrm{C}}$ & 1.37 & GCN 9956 \\
GRB 091127 $^{\mathrm{D}}$ & $18.9 \pm 0.1$ & 0.49 & GCN 10238 \\
\hline
\end{tabular}

${ }^{\mathrm{A}}$ GRB 081118 is one of the most distant transients imaged with a video camera (the Adirondack Stellacam).

${ }^{\mathrm{B}}$ Possibly a short GRB.

${ }^{\mathrm{C}}$ Based on $R$-band magnitudes of USNO catalogue stars.

${ }^{\mathrm{D}}$ GRB with unusually slow-decaying OA.

- GRB 081118: This was the first optical transient imaged by the ZT. Imaging started $1.96 \mathrm{~h}$ post-Swift burst trigger, using an unfiltered, integrating $\mathrm{CCD}$ video camera, and continued for $1 \mathrm{~h}$. The OA candidate reported in GCN 8529 was seen in a selected stack of $40 \times 5 \mathrm{~s}$ exposures, though it was near the detection limit. Using nearby USNO-B1 stars, the OA magnitude was found to be $20.9 \pm 0.5$ (GCN 8675). Confirmations of this OA candidate were made by the $2.2 \mathrm{~m}$ GROND telescope and the VLT; a VLT spectroscopic redshift of $z=2.58$ was determined $8 \mathrm{~h}$ after the ZT observation.

- GRB 090205: This was a late-time observation; imaging started $17.6 \mathrm{~h}$ post-Swift trigger, with the unfiltered Andor camera, and continued for $4460 \mathrm{~s}$. In a $1100 \mathrm{~s}$ stacked image, a candidate OA with $23.2 \pm 0.3$ white magnitude was marginally detected $(\mathrm{SNR}=3.3$ ) at the enhanced XRT position reported in GCN 8885 . This OA magnitude was estimated using nearby USNO-B1 stars (GCN 8976). As the source was near the detection limit, the same field was imaged 2 weeks later: the candidate OA was not detected, which is consistent with a fading GRB as a possible cause of this transient.

- GRB 090313: Imaging of the field reported in GCN 8980 started $4.26 \mathrm{~h}$ post-Swift trigger, with significant sky brightness from the Moon. The OA decayed from $18.02 \pm 0.08$ to $18.84 \pm 0.12$ in $8500 \mathrm{~s}$ (GCN 8996).

- GRB 090509: Imaging of the field reported in GCN 9325 started $8 \mathrm{~h}$ post-Swift trigger; $48 \times 5 \mathrm{~s}$ exposures were made over $14 \mathrm{~min}$. Due to sky brightness from a full Moon, no candidate OA was found at the GROND position (GCN 9326), nor at the BAT-refined position (GCN 9349), with an upper limit of $R=17.6$ estimated from nearby USNO-B1 stars (GCN 9363).

- GRB 090516: Imaging started $275 \mathrm{~min}$ post-Swift trigger, and continued for $131 \mathrm{~min}$. An OA candidate was found within the XRT error circle, and it faded from $18.9 \pm 0.5$ to $20.26 \pm 0.14$ over $21 \mathrm{~min}$ (GCN 9380).

- GRB 090927 - a possible short GRB: Imaging of the field reported by Swift/UVOT (GCN 9946) started $110 \mathrm{~min}$ post-Swift trigger, and continued for $50 \mathrm{~min}$

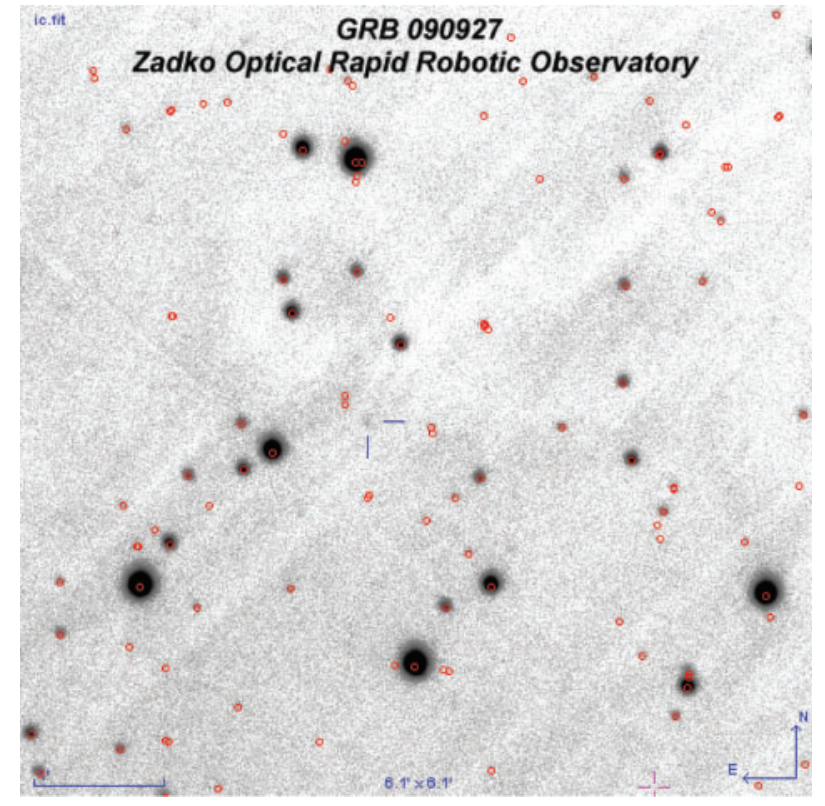

Figure 6 GRB 090927 optical afterglow, shown by the pointer marks near the image centre. Red circles are known stars from the NOMAD1 catalog. This ZT image is a sum of $2 \times 180 \mathrm{~s}$ exposures taken $2.6 \mathrm{~h}$ post-Swift trigger. Stellar images have $\theta_{\mathrm{FWHM}}=$ 1.8 arcsec. The afterglow at this time had $R=20.0 \pm 0.5$ (image processed by TAROT collaboration).

in piloted robotic mode. A fading source was found within the XRT error circle - see Figure 6. Preliminary photometry of this OA candidate gave $R$-band magnitudes of: $19.8 \pm 0.5$ (540 s exposure) and $20.0 \pm 0.5$ $(360 \mathrm{~s})$, at post-burst times of 126 and $158 \mathrm{~min}$, respectively (GCN 9956).

- GRB 091127 - with a slowly-decaying OA: This was a bright $15.0 \mathrm{mag}$ source which triggered after dawn but had an unusually slow decay time. Using a Pentax K200D digital SLR camera mounted at the Cassegrain focus, late-time imaging of the field reported in GCN 10191 started $18.5 \mathrm{~h}$ post-Swift trigger and continued for $28 \mathrm{~min}$; during this time the Moon set and weather conditions were good. A faint source was found at the OA candidate location reported in GCN 10199. Photometry on a co-added sum of 4 CCD images (of $806 \mathrm{~s}$ total exposure time) gave an $\mathrm{OA}$ magnitude of $18.9 \pm 0.1$ (GCN 10238).

\section{Future Development}

\subsection{Instrument Upgrade}

The Guide Acquire Module (GAM) supplied with the DFM telescope places the optimal image plane too close to the current optical mounting plate to allow a large filter wheel to be placed in front of the current Andor camera. There are several solutions to this problem, depending on available funds; a particularly attractive one would be to replace the GAM with a new optical mounting structure, to ensure that there is space for mounting the filter wheel, electronic focuser, and Andor CCD camera. 
The new design will allow for three optical paths: for filtered CCD imaging, an integrating video camera and a spectrograph.

Subject to funding, a new CCD camera, filter wheel, and filter system will be installed. One of the imagers being considered is the Fairchild Peregrine 486 CCD camera which can be matched to the image focal plane of the existing optical mount. It has a $61.44 \times 61.44 \mathrm{~mm}$ chip, with an imaging FOV of $1^{\circ}$ or 7 times the area of the existing camera; by significantly increasing the survey speed, this camera would extend the science capabilities of the ZT in the field of transient searches.

\subsection{ZT Science Linkages}

ZT research is tightly linked to several existing and upcoming international programs in transient astronomy. The ZT team is part of the TAROT collaboration, which already uses two other very rapid response telescopes (in France and Chile). We are working closely with the GW astronomy community (the LIGO Scientific Collaboration) to use the ZT for optical follow-ups of candidate $\mathrm{GW}$ events, and to survey galaxies within the LIGO sensitivity horizon for coincident optical-GW transients. Our TAROT collaboration is also working with the the Antares deep-sea neutrino telescope to follow up on possible neutrino events. These projects are at the frontier of multi-messenger astronomy, and it is very timely for the ZT to play a role when these projects are just starting.

The U.S. GW observatory LIGO started searching for GW transient signals, with unprecedented sensitivity, in late 2009 (Abbott et al. 2007). Despite the detection of significant numbers of candidate GW transient events in LIGO data in recent science runs, the SNR of these correlated signals is relatively small. It is difficult to confirm that these events are astrophysical in origin, rather than environmental (Fox et al. 2005). However, the association of such an event with an astrophysical transient observed in the optical would dramatically improve the confidence of GW detection. The ZT, with our partners in the TAROT collaboration and other transient telescopes, will be testing and developing procedures to search for coincident GW and optical transients.

The Australian SKA Pathfinder (ASKAP), currently under construction, is a revolutionary new design of radio telescope, capable of monitoring $30 \mathrm{deg}^{2}$ of sky instantaneously, forming images with $10 \mathrm{arcsec}$ resolution (Johnston et al. 2008). ASKAP will have both wide sky coverage and high sensitivity, plus the ability to locate transient radio sources to high precision. However, for fast radio transients that appear and disappear on timescales as short as a millisecond, searches will initially be incoherent (i.e. uncorrelated); this will only provide low-resolution positional information, although offline postprocessing of the raw data can also provide highresolution positions. Incoherent detection will provide ASKAP triggers with positional accuracy of $1^{\circ}$ which is well-matched to the capability of the ZT once it is upgraded to achieve a $1^{\circ} \mathrm{FOV}$. The proximity of the ZT to ASKAP also provides a unique advantage in the followup of short-lived ASKAP radio transients.

\subsection{Remote Access Astronomy in Education}

The ZT has initiated a novel public outreach program that allows small groups to obtain CCD images on site for a few hours every month. This program is part of a broader science outreach project. Furthermore, projects are being developed that will allow students to participate in ZT science, and to design and implement projects which will allow them to make useful scientific contributions. The project list is long, but includes:

- Minor planet and NEO recovery and discovery.

- Supernova surveys, discovery and follow-up.

- GRB and other transient event follow-up.

As the global awareness of the importance of astronomy as a context for teaching science continues to increase, educators are now able to re-engage in observational astronomy through the use of remote access to robotic telescopes. In parallel with this trend, wide-field instruments are generating a wealth of data directly available to the public for analysis. There are very practical reasons for this, namely that the huge data volumes make it difficult to search for faint optical transients. This represents an opportunity for science educators to tap into at relatively small cost, with the benefit of contributing to real science.

The ZT is primed to take advantage of these opportunities in education, training and outreach. The ZT observatory control system, using the TAROT robotic system, allows science data to be acquired remotely using a webbased interface. Our French collaborators have successfully used this system for education and outreach. The ZT will act as a node in a distributed network of robotic telescopes, allowing the opportunity for students in Europe to acquire image data from the ZT. Conversely, local students can acquire data from the other networked telescopes in France and Chile.

An education research project is being developed, as part of an ARC Linkage grant, aimed at measuring the effectiveness of student learning via remote access astronomy. With astronomy as the context, this will provide valuable data on how different modes of learning science influence students' choices to continue science education from high school to tertiary level.

\section{Acknowledgments}

D. M. Coward is supported by ARC grants DP 0877550 , LP 0667494, and by the University of Western Australia (UWA). The ZT facility is managed by the School of Physics, UWA, the International Centre for Radio Astronomy Research (ICRAR), and the Australian International Gravitational Research Centre (AIGRC). We also acknowledge the Centre for Learning Technology (CLT) at UWA, and the Western Australian Department of Education and Training (DET), for supporting a science training and outreach program. 


\section{References}

Abadie, J. et al. (LIGO Scientific Collaboration and the VIRGO Collaboration), 2010, LSC Document LIGO-P0900125, e-print (arxiv:1003.2480v2 [astro-ph.HE])

Abbott, B. et al., 2007, PhRvD, 76, 062003

Andor Technology PLC, 2007, iKon-L DW436-BV Technical Document (Belfast, N. Ireland: Andor Technology PLC)

Barbieri, C., 2007, Fundamentals of Astronomy (Boca Raton FL, USA: CRC Press, Taylor \& Francis), 292

Bertin, E. \& Arnouts, S., 1996, A\&AS, 117, 393

Bourez-Laas, M., Vachier, F., Klotz, A., Damerdji, Y. \& Boër, M., 2008, Proc. SPIE, 7019, 701918

Coward, D. M., Guetta, D., Burman, R. R. \& Imerito, A., 2008, MNRAS, 386, 111

Fox, D. B. et al., 2005, Nature, 437, 845
Johnston, S. et al., 2008, ExA, 22, 151

Klotz, A., Boër, M., Eysseric, J., Damerdji, Y., Laas-Bourez, M., Pollas, C. \& Vachier, F., 2008a, PASP, 120, 1298

Klotz, A., Vachier, F. \& Boër, M., 2008b, AN, 329, 275

Klotz, A., Gendre, B., Atteia, J. L., Boër, M., Coward, D. M. \& Imerito, A. C., 2009, ApJ, 697, L18

McLean, I. S., 2008, Electronic Imaging in Astronomy: Detectors and Instrumentation (2nd ed; Chichester, UK: Springer-Praxis Publ. Ltd.), 46

Saha, A., Dolphin, A. E., Thim, F. \& Whitmore, B., 2005, PASP, 117,37

Sathyaprakash, B. S. \& Schutz, B. F., 2009, Living Reviews in Relativity, 12(2), 99-102 (arxiv:0903.0338v1 [gr-qc])

Zombeck, M. V., 1990, Handbook of Space Astronomy \& Astrophysics (2nd ed; Cambridge, UK: Cambridge Univ. Press), 100 